\title{
Unprecedented and Readily Tunable Photoluminescence from Aliphatic Quaternary Ammonium Salts
}

Saixing Tang, Zihao Zhao, Tianjia Yang, Yunzhong Wang, Xiaohong Chen and Wang Zhang Yuan*

School of Chemistry and Chemical Engineering, Frontiers Science Center for Transformative Molecules, Shanghai Key Lab of Electrical Insulation and Thermal Aging, Shanghai Jiao Tong University, No. 800 Dongchuan Rd., Minhang District, Shanghai 200240, China

E-mail:wzhyuan@sjtu.edu.cn

\begin{abstract}
Compounds bearing aliphatic amines could be emissive under proper conditions. Their ionized counterparts, quaternary ammonium salts (QASs), which are widely used as phase-transfer catalysts, ionic liquids, disinfectants and surfactants, however, are known as luminescence quenchers and thought to be nonemissive. Here we report the unprecedented intrinsic fluorescence-phosphorescence dual emission from a diversity of QASs, which can also be finely regulated by excitation wavelength, alkyl chain length, counter ions, mechanical stimuli, etc. The bright photoluminescence along with distinct afterglow and tunable multicolor emissions enables advanced multimode anticounterfeiting for QAS solids. This finding refreshes the cognition of QASs and would inspire emerging applications by utilizing their intrinsic luminescence. Furthermore, it opens opportunities for the investigation of QAS related processes and functions in a photophysical approach and affords strong implications for the fabrication of novel nonconventional luminophores.
\end{abstract}

Keywords: Aliphatic quaternary ammonium salts, Charge transfer, Clustering-triggered emission, Multitunable photoluminescence, Nonconventional luminophores 
Recently, nonconventional luminophores without remarkable conjugations but with electron-rich auxochromes like amine, cryano, aimde and heteroatoms, have been highly valued for their such merits as facile synthesis, environmental friendliness and excellent biocompatibility. ${ }^{[1-8]}$ Thanks to the enrichment of $\mathrm{n}$ electron-rich moieties (e.g. $\mathrm{OH}, \mathrm{NH}_{2}, \mathrm{C}=\mathrm{O}, \mathrm{CN}$ ) and effective clustering, besides fluorescence, unique phosphorescence and even persistent room temperature phosphorescence (p-RTP) can also be acquired from the aggregates (e.g. crystals and films) of nonconventional luminophores, owing to the promoted spin-orbit coupling (SOC), narrowed singlet-triplet energy gaps and thereby faciliated intersystem crossing (ISC) processes. ${ }^{[8-15]}$ Among different nonconventional luminophores, ${ }^{[16-}$ 21] aliphatic amines are mostly investigated, ${ }^{[3-5,16-18]}$ with polyamidoamine (PAMAM) as the representative. ${ }^{[3,4,17]}$ Intensive endeavors have been made to decipher their emission mechanism involving various concerns on $\mathrm{pH}$, terminal group and architecture. ${ }^{[3,4,18]}$ Thereinto, oxidation is widely believed as the origin of the emission, in which tertiary amines are believed as the prerequisite. ${ }^{[4,17,22]}$ These early perceptions render quaternary ammonium salts (QASs), which are also utilized as luminescence quenchers, ${ }^{[23-25]}$ are regarded as nonluminescent. ${ }^{[17]}$

Although the oxidation mechanism has long influenced the research on nonconventional luminophores, particularly those of aliphatic amines, exciting progresses have been achieved in other systems. ${ }^{[5,6,26-28]}$ Furthermore, bright emission was observed from PAMAM and PEI aggregates even without oxidation, ${ }^{\text {[29] }}$ which strongly excludes oxidation as the origination of emission in such systems. These luminescent phenomena, however, can be rationalized by the clustering-triggered emission (CTE) mechanism, which emphasizes the clustering of nonconventional chromophores, extended electron delocalization and conformation rigidification. ${ }^{[29-31]}$

In this contribution, we report our new finding of the unexpected but widespread intrinsic photoluminescence (PL) from aliphatic QASs (Fig 1). Furthermore, tunable PL and p-RTP are readily accessed through changing the excitation wavelength $\left(\lambda_{\mathrm{ex}}\right)$, mechanical grinding and adjusting the alkyl chain length, counterions (i.e. $\mathrm{Cl}^{-}, \mathrm{Br}^{-}$, and $\mathrm{I}^{-}$) and temperature. To the best of our knowledge, these are the first examples of highly luminescent QASs, whose crystal efficiency $\left(\Phi_{\mathrm{c}}\right)$ and p-RTP lifetime $\left(<\tau>_{\mathrm{p}}\right)$ are of up to $14.7 \%$ and $761.9 \mathrm{~ms}$, respectively. Single crystal structure analysis and theoretical results collaboratively indicate that such unique emission is highly associated to the clustering of ion pairs of QASs with distinct through-space conjugation (TSC) and charge transfer (Fig 1) characteristics. 


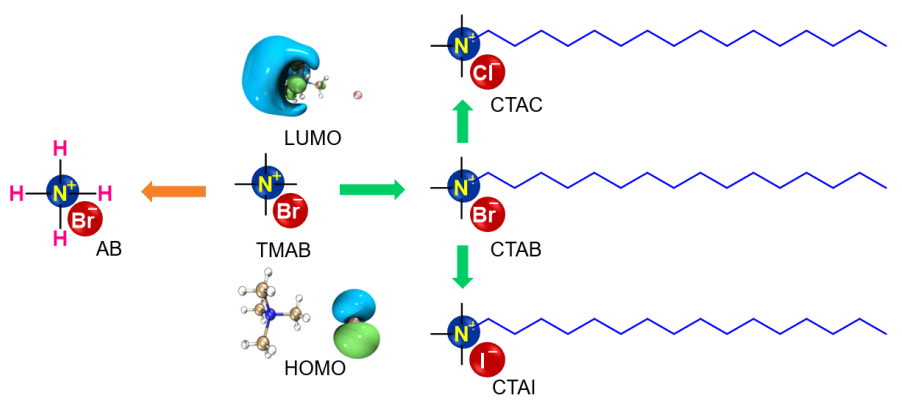

Figure 1. (a) QASs studied herein and HOMO/LUMO electron densities of single TMAB molecule.

\section{Solid PL properties of QASs with bromide counterion}

We initially checked the PL of single crystals of ammonium tetramethyl bromide (TMAB), one of the most simplest QASs (Fig S1-S3, Table S1). Interestingly, the crystals demonstrate bright bluish-white or blue PL under varying UV irradiations (Fig 2a). PL spectra show the maxima at 402, 439 and $445 \mathrm{~nm}$ with $\lambda_{\text {ex }}$ of 254, 312 and $365 \mathrm{~nm}$, respectively, indicating their excitation-dependent feature (Fig 2b). Furthermore, with a delay time $\left(t_{\mathrm{d}}\right)$ of $1 \mathrm{~ms}$, distinct profiles with maxima at 515/650, 515 and 476/530 $\mathrm{nm}$ in response to different $\lambda_{\mathrm{ex}} \mathrm{s}$ (Fig $2 \mathrm{~b}$ ) were recorded. These delayed emissions are assignable to RTP, which is further verified by the various lifetimes of several milliseconds (Fig S4). For comparison, PL properties of ammonium bromide (AB) crystals were also investigated. In stark contrast to the bright bluish-white emission of TMAB crystals with $\Phi_{\mathrm{c}}$ of $14.6 \%$, AB crystals are nearly nonemissive with an extremely low $\Phi_{\mathrm{c}}$ of $0.2 \%$. Such emission, however, can be apprently brightened with distinct afterglow upon freezing to $77 \mathrm{~K}$ (Fig S5). These results, on one hand, verify the general intrinsic light emission from the ammonium compounds, on the other hand, demonstrate the opportunity to tune their PL through the alkyl chain engineering. ${ }^{[32]}$ In that scenario, cetyltrimethylammonium bromide (CTAB), a well-known surfactant, with a much longer alkyl chain was further investigated. The long alkyl chain might regulate molecular stacking, noncovalent interactions and consequent PL properties. ${ }^{[32]}$ Intriguingly, upon irradiation, other than prompt blue emission, distinct blueish-green to greenish-yellow afterglow is observed from CTAB crystals, which is absent in their TMAB counterpart (Fig 2a). In addition, excitation-dependnet PL is also noticed for CTAB crystals. As depicted in Fig $2 b$, when the $\lambda_{\text {ex }}$ changes from 254 to 312 and then to $365 \mathrm{~nm}$, the emission maximum of CTAB crystals gradually shifts from $356 / 416$ to 415 and then to $462 \mathrm{~nm}$, accompanying the delayed emission ( $t_{\mathrm{d}}=1 \mathrm{~ms}$ ) maxima at 490, 530 and $542 \mathrm{~nm}$, respectively. Meanwhile, the Commission Internationale de l'Éclairage (CIE) coordinate shifts from $(0.26,0.33)$ to $(0.30,0.38)$ and then to $(0.31,0.35)$ (Fig S6a), consistent with the naked-eye observation. Such delayed emission should also be ascribed to the crystal p-RTP, which illustrates various long lifetimes of up to $761.9 \mathrm{~ms}$ (Fig 2e), indicating the presence of heterogeneous emissive species accountable for the excitation-dependent emission. 

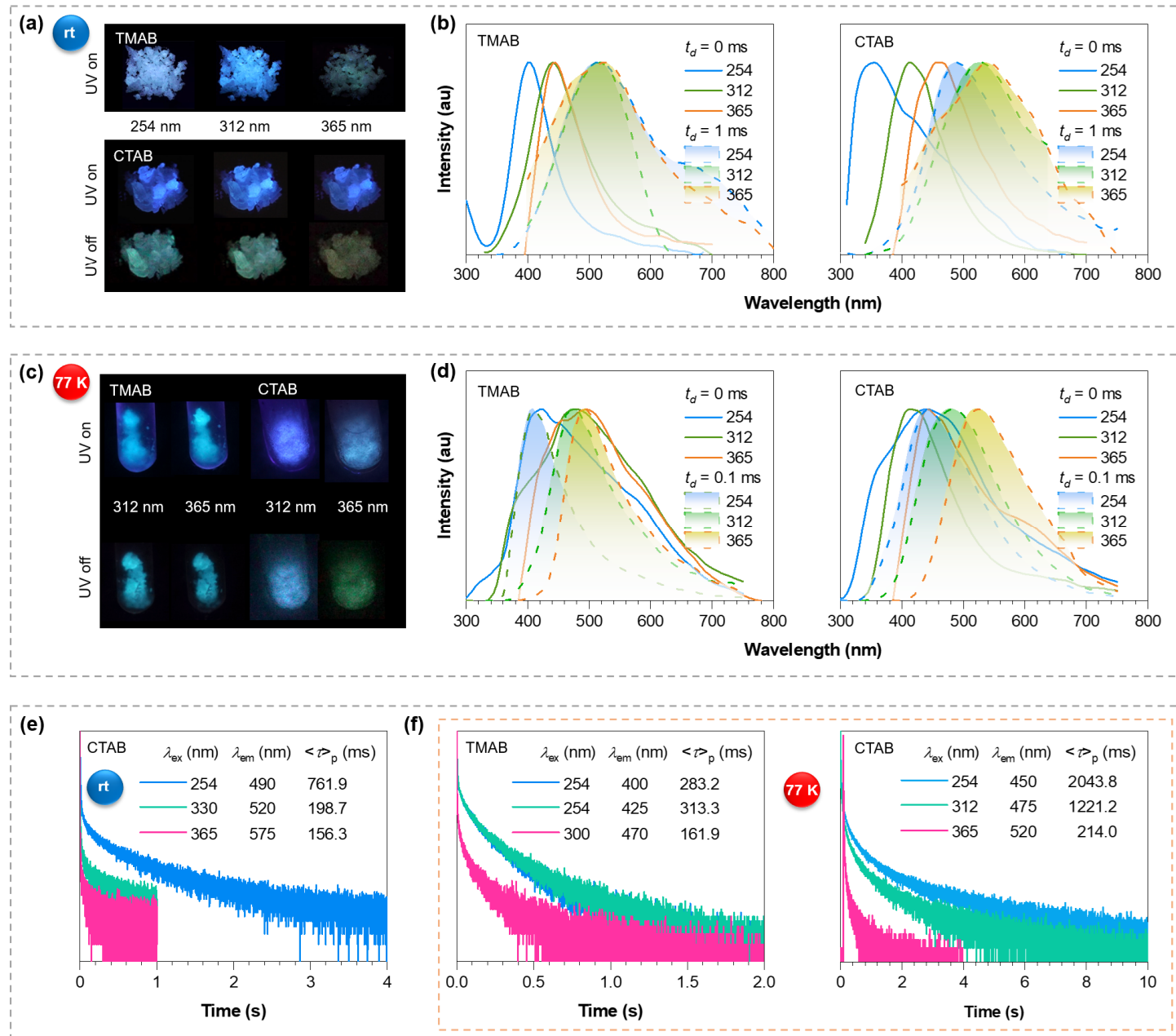

Figure 2. Photophysical properties of TMAB and CTAB crystals. (a,c) Photographs taken under different UV lights or after the stop of UV irradiations and $(b, d)$ prompt and delayed $\left(t_{\mathrm{d}}=1 \mathrm{~ms}\right)$ emission spectra with different $\lambda_{\mathrm{ex}} \mathrm{S}$ for TMAB and CTAB crystals at $(\mathrm{a}, \mathrm{b})$ room temperature and $(\mathrm{c}, \mathrm{d}) 77 \mathrm{~K}$. (e,f) Time-resolved decay profiles of the afterglows for $\mathrm{CTAB}$ and TMAB crystals at (e) room temperature and (f) $77 \mathrm{~K}$.

Upon cooling to $77 \mathrm{~K}$, the PL of TMAB and CTAB crystals gets enhanced with broadened prompt spectra (Fig 2c,d). Furthermore, green phosphorescence afterglow $\left(<\tau>_{\mathrm{p}} \mathrm{s}: 161.9 \sim 313.3 \mathrm{~ms}\right)$ is enabled from TMAB crystals due to rigidified conformations and subsequently diminished molecular motions at cryotemperature (Fig 2f). Meanwhile, compared to those at room temperature, significantly blue-shifted phosphorescence maximum (from 515 to $409 \mathrm{~nm}$ ) is observed under $254 \mathrm{~nm}$ UV excitation at $77 \mathrm{~K}$, accompanying narrowed full-width at half-maximum (FWHM, from 163 to $119 \mathrm{~nm}$ ). Such phenomenon suggests the more intensified emission at much bluer regions associated with less delocalized emissive clusters. Similarly, for CTAB crystals, with $\lambda_{\text {ex }}$ changing from 254 to $365 \mathrm{~nm}$, boosted and prolonged afterglows from blue $(442 \mathrm{~nm})$ to green $(525 \mathrm{~nm})$ are noticed at $77 \mathrm{~K}$, along with different $<\tau>{ }_{\mathrm{p}} \mathrm{s}$ of up to $\sim 2 \mathrm{~s}$ (Fig 2f) and correspondingly evolved CIE coordinate from $(0.20,0.21)$ to $(0.32,0.45)$ (Fig S6b). Such more obvious $\lambda_{\text {ex }}$-dependent emission again verifies the presence of miscellaneous emissive species, 
whose excitons can be further stabilized at cryotemperature, thus resulting in enhanced PL and much wider color tunability (Fig S7).
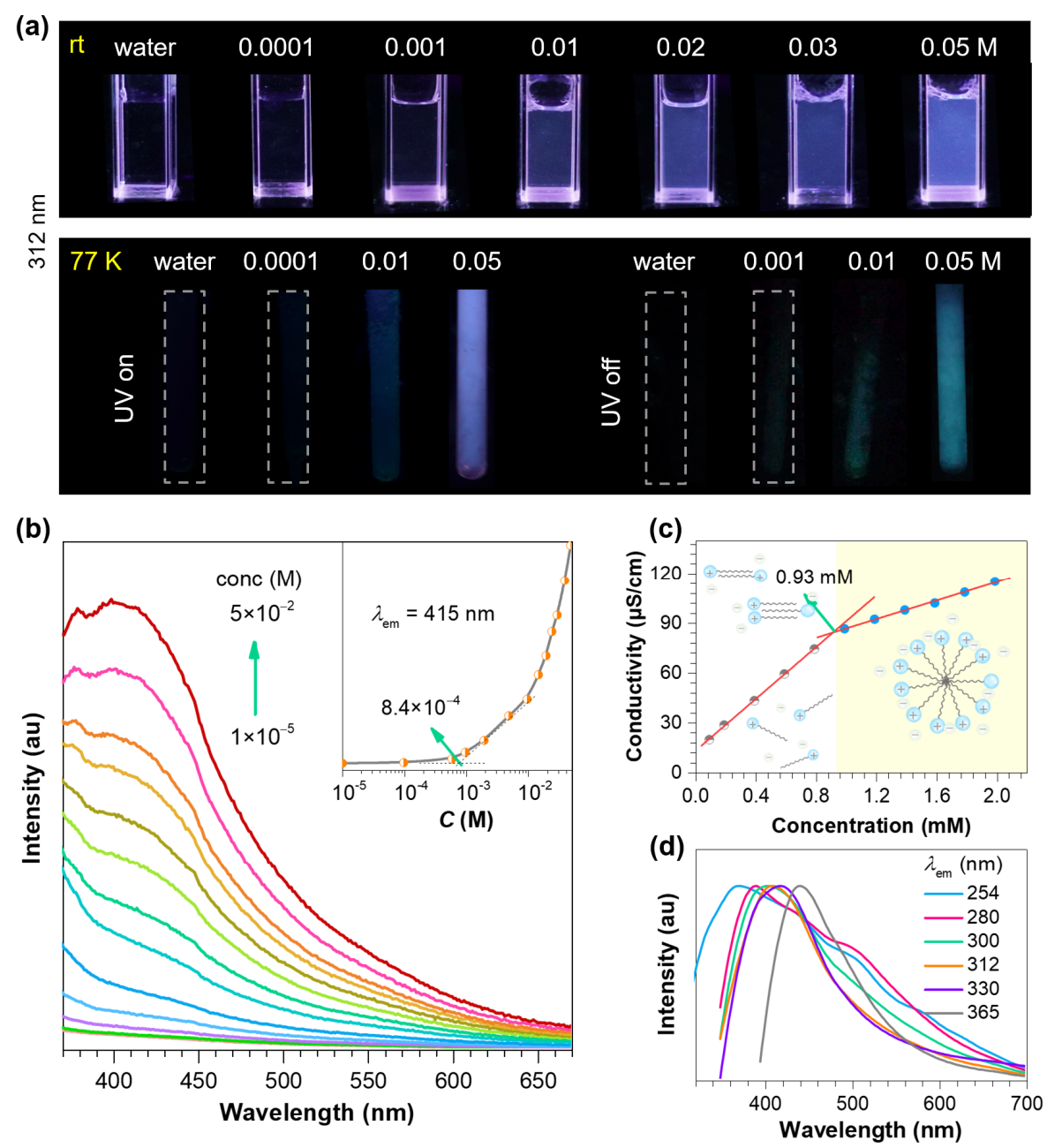

Figure 3. Photophysical properties of aqueous CTAB solutions. (a) Photographs of aqueous CTAB solutions at different concentrations taken under $312 \mathrm{~nm}$ UV light or after ceasing the irradiation at room temperature or $77 \mathrm{~K}$. (b) PL spectra $\left(\lambda_{\mathrm{ex}}=312 \mathrm{~nm}\right)$ and the peak intensity $(415 \mathrm{~nm}$, inset) of CTAB aqueous solutions with different concentrations. (c) Demonstration of the conductivity and structure evolution of $\mathrm{CTAB}$ aqueous solutions at different concentrations. (d) PL spectra of $5 \times 10^{-2} \mathrm{M} \mathrm{CTAB}$ aqueous solution at different $\lambda_{\mathrm{ex}} \mathrm{s}$.

\section{Photophysical properties of CTAB aqueous solutions}

To acquire more insights into the emission, we investigated the photophysical properties of CTAB aqueous solutions. Dilute solutions $\left(e . g .10^{-4} \mathrm{M}\right)$ show no visible emission even at cryogenic state, with PL signals comparable to those of the solvent being recorded (Fig 3a,b), which strongly suggests the nonluminescent nature of individual CTAB molecules. With increased concentrations, remarkable blue PL is noticed at $0.01 \mathrm{M}$ (Fig 3a), and the PL intensity is progressively boosted until a concentration of $5 \times 10^{-2} \mathrm{M}$ (Fig 3b), above which the PL starts to decrease due to the solid precipitation. Additionally, at 
$77 \mathrm{~K}$, enhanced PL together with cyan afterglows are visualized for concentrated solutions (Fig 3a), thanks to the clustering of ion pairs and seriously stiffened conformations. Notably, emerging shoulders at 393 and $415 \mathrm{~nm}$ are found at the concentration of $8 \times 10^{-4} \mathrm{M}$, and an inflexion point at $8.4 \times 10^{-4} \mathrm{M}$ is derived from the plot of PL peak intensity versus concentration. These points highly approach the critical micelle concentration $(\mathrm{CMC})$ of CTAB $\left(9.3 \times 10^{-4} \mathrm{M}\right)$ determined by the electrical conductivity method (Fig 3c), indicative of an alternative PL technology for the measurement of CMC of QAS based surfactants. Notably, as illustrated in Fig. 3c, only individual molecules or small aggregates are present in solutions with concentrations below $\mathrm{CMC}$, which are virtually nonluminescent even at cryotemperature. In contrast, beyond $\mathrm{CMC}$, noticeable emission can be detected at room temperature. These results definitely suggest the crucial role of aggregation for the intrinsic emission of QASs. Specifically, for the concentrated solutions (e.g. $0.05 \mathrm{M}$ ), significant $\lambda_{\text {ex }}$-dependent PL with diverse peaks at $371 / 391 / 405 / 410 / 420 / 440 \mathrm{~nm}$ (Fig 3d) is recorded, indicative of the concurrence of multiple emissive species, as further supported by their varying fluorescent lifetimes (Fig S8).

\section{Counterions regulated emission}

The impressive PL properties of TMAB and CTAB illustrate that QASs could be competitive candidates for the generation of efficient (p-)RTP, which is currently extensively studied in aromatics. ${ }^{[33-}$ ${ }^{44]}$ In view of the strong Coulombic interaction between halogen counterions $\left(\mathrm{X}^{-}\right)$and positively charged quaternary nitrogen moieties $\left(\mathrm{N}^{+}\right)$, the heavy halogen ions should play an important role in the formation of clustered chromophores. Meanwhile, they can also considerably enhance the SOC and subsequent ISC processes due to the clustering effect ${ }^{[45]}$ and the well-known heavy atom effect ${ }^{[46,47]}$. Consequently, the PL and RTP of QASs could be effectively regulated via simple counterion exchange. Bearing this in mind, we further investigated CTAC and CTAI with counterions of $\mathrm{Cl}^{-}$and $\mathrm{I}^{-}$, respectively. Similar to CTAB, both crystals exhibit bright PL and distinct p-RTP with apparent $\lambda_{\text {ex }}$-dependent property (Fig 4 and Fig S9-S12). Different crystals, however, demonstrate various efficiencies and p-RTP lifetimes. While CTAC crystals show the highest $\Phi_{\mathrm{c}}$ of $14.7 \%$, which is about twice that of CTAB crystals $(7.2 \%)$, CTAB crystals enjoy the longest $\left\langle\tau>_{\mathrm{p}}\right.$ of $716.9 \mathrm{~ms}$ (Fig 4c,d). Meanwhile, the PL and p-RTP colors are also variable with different counterions, owing to the change in relative intensities in blue and red regions (Fig 2a and Fig 4). Impressively, CTAI crystals glow orange-red under $254 \mathrm{~nm}$ UV light, which turns into greenish blue and further changes to blue while irradiated by 312 and $365 \mathrm{~nm}$ UV lights (Fig 4a). Concretely, with 254 nm UV excitation, CTAI crystals exhibit the exceptional orange-red PL with the peak and shoulders at 615 and 360/458 nm, whereas CTAC and CTAB crystals emit at 360 and $356 \mathrm{~nm}$ (Fig 4b), respectively. Moreover, distinct bluish-green (479 nm, $558.6 \mathrm{~ms})$, green $(440 / 478 / 517 \mathrm{~nm})$ and yellow $(563 \mathrm{~nm}) \mathrm{p}$ RTP afterglows are visualized for CTAI crystals after ceasing the 254, 312 and $365 \mathrm{~nm}$ UV irradiations (Fig 4a,d and Fig S9), respectively. 
To probe the nature of CTAI crystal emissions, their lifetimes were measured. For the prompt emission at 360 and $630 \mathrm{~nm}$, lifetiems of 2.0 and $290.8 \mathrm{~ns}$ (Fig 4d and Fig S13) are obtained, suggesting their prompt fluorescence and RTP nature, respectively. Compared to CTAI crystals, CTAC crystals show generally redshifted p-RTP maximum with a much wider adjustable range from 493-540 nm to 479-563 $\mathrm{nm}$ while excited at intervals from 254 to $365 \mathrm{~nm}$ (Fig 4b), along with decreased $\Phi_{\mathrm{c}}$ and increased $\left\langle\tau>_{\mathrm{p}}\right.$ (Fig 4c,d). These results, on one hand, testify the widespread intrinsic emission of QASs, on the other hand, well corroborate the feasibility to regulate PL and (p-)RTP of QASs through facile counterion exchange.

(a)
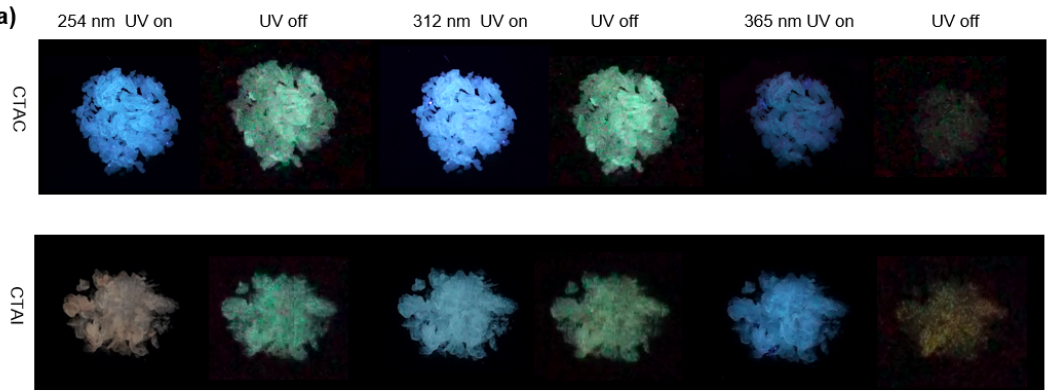

(c)

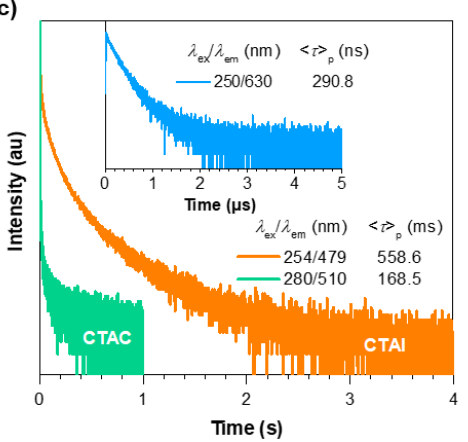

(d)

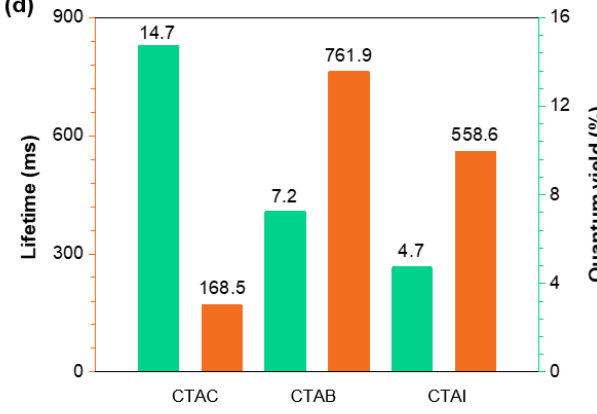

(b)

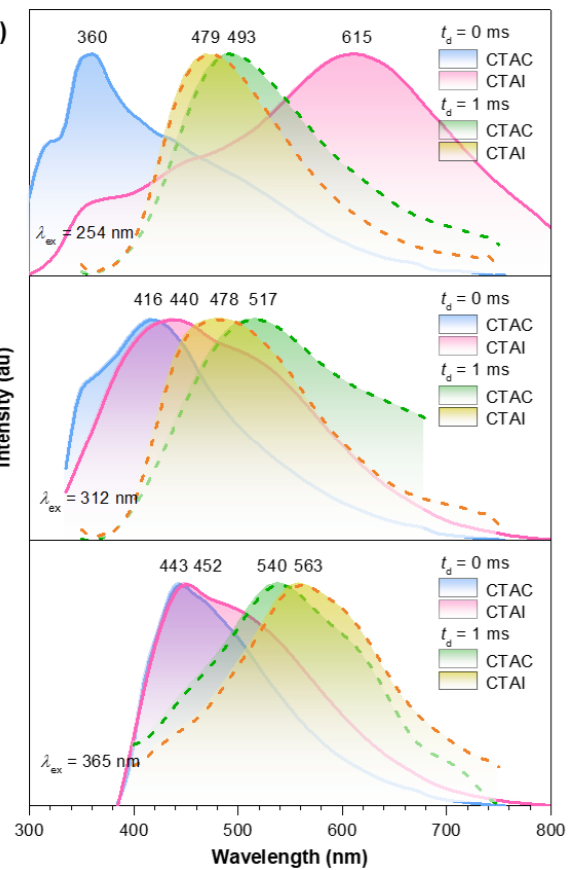

Figure 4. PL properties of CTAC and CTAI crystals. (a) Photographs of CTAC and CTAI crystals taken under 254, 312 and $365 \mathrm{~nm}$ UV lights or after ceasing the UV irradiations. (b) Prompt and delayed $\left(t_{\mathrm{d}}=1 \mathrm{~ms}\right)$ emission spectra of CTAC and CTAI crystals with different $\lambda_{\mathrm{ex}} \mathrm{s}$. (c) p-RTP lifetime profiles of the CTAC crystals under excitation. Nanosecond ( $\left.\mathrm{ns}, \lambda_{\mathrm{ex}}=250 \mathrm{~nm}\right)$ and millisecond $\left(\mathrm{ms}, \lambda_{\mathrm{ex}}=312 \mathrm{~nm}\right)$ scale lifetimes for CTAI crystals. (d) Quantum yields and p-RTP lifetimes for CTAC, CTAB and CTAI crystals.

\section{Unique organe-red PL of CTAI crystals and emission mechanism understanding}

It is so extraordinary that CTAI crystals can demonstrate orange-red RTP emission under $254 \mathrm{~nm}$ UV irradiation. To explore more details together with the corresponding emission mechanism, we further investigated the emission with $\lambda_{\mathrm{ex}}$ from 220 to $260 \mathrm{~nm}$. While the emission covers a wide range of $350 \sim 800 \mathrm{~nm}$, two main emission maxima at blue $(\sim 438,480 \mathrm{~nm})$ and orange-red $(\sim 625,636 \mathrm{~nm})$ regions are noticed with variable relative intensities (Fig 5a). And the overall luminescent color undergoes blue to orange and then to blue again (Fig S14), suggestive of highly complex photophysical processes. The 
readily tunable PL of these QASs can be rationalized by a charge transfer process from halogen anions to clustered chromophores, in synergy with energy transfer occurring between diversified clusters with different electronic delocalizations and energy levels.

To gain deeper insights into the emission behaviors of these QASs, we determined their single crystal structures. Taking CTAI as an example, the ion pairs are close to each other through ionic linkage, and the alkyl chains are arranged in neat layers, which facilitates the extensive electron communication amongst ionic clusters. Abundant intermolecular dihydrogen bonds $(\mathrm{C}-\mathrm{H} \cdots \mathrm{H}-\mathrm{C})$ and halogen contacts $(\mathrm{C}-\mathrm{H} \cdots \mathrm{I})$ are also found in CTAI crystals (Fig S15d), which help connecting quaternary ammonium cations and iodine counterions and diminishing the molecular motions, thus leading to rigidified conformations and restrained nonradiative dissipations. It is also noted that one quaternary ammonium cation is surrounded by four or five conterions (Fig 5b and Fig S16), which is beneficial to electron delocalization and conformation rigidification owing to the extensive ionic network.

We also conducted theoretical calculations to explore the electronic structure and energy levels of the compounds. For better clarity, monomer, dimer and trimer of the compounds were utilized. As depicted in Fig 5c, while the energy densities of HOMOs are mainly distributed around single iodine anions, those of LUMOs are mostly decolcalized on quaternary ammonium cations, demonstrating conspicuous charge transfer and TSC characteristics. These results further support our assumption that clustered ion pairs with distinct charge transfer are associated to the emission of QASs. When clustered together, the throughspace electron delocalization enriches the energy levels and narrows the energy gap between ground states and excited states (Fig 5d), thus beneficial to exciation and emission. Furthermore, the calculated energy gaps between lowest siglets and triplets are small (Fig 5d), thus enabling efficient ISC process and consequent phosphorescence emission.

Concerning the results of the experiments and theoretical calculation, the emission can be rationalized by the CTE mechanism, ${ }^{[29-31]}$ namely clustered ion pairs with extended through-space electron delocalization and sufficiently stiffen conformations are correlated to the emission of QASs in concentrated solutions and crystals. Besides, the remarkable overlap between UV absorption and PL emission of CTAI crystals indicate some energy transfer processes may readily occur among the clusters (Fig S17), therefore, the remarkable orange-red emission is attributable to RTP with tandem charge transfer and energy transfer processes amongst diversified clusters (Fig 5e). Furthermore, the diverse molecular packings and various charge transfer features of different QASs might be responsible for their distinct luminescent properties. 


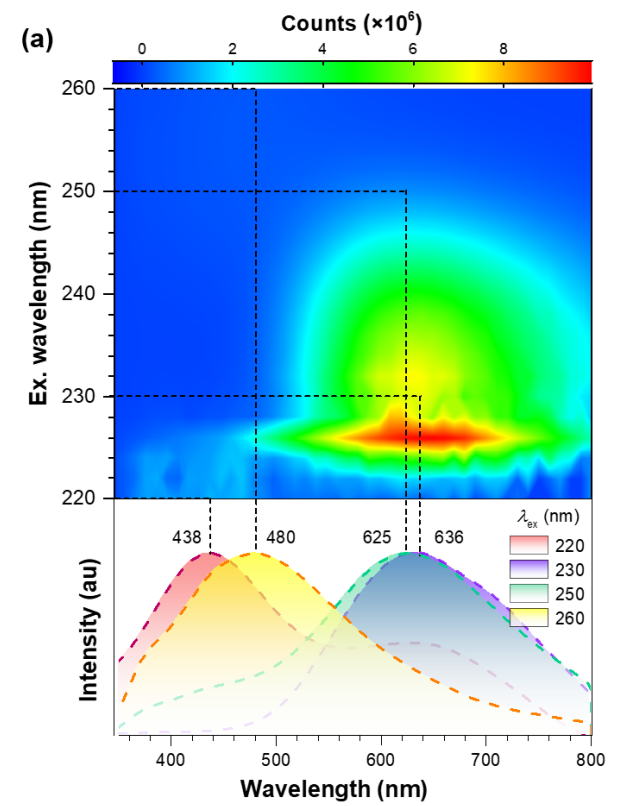

(b)

(c)

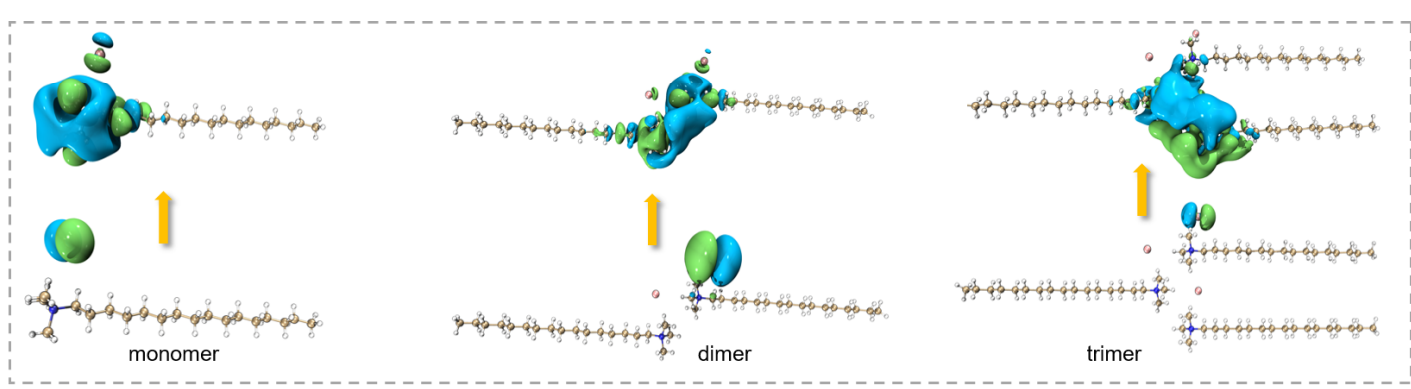

(d)

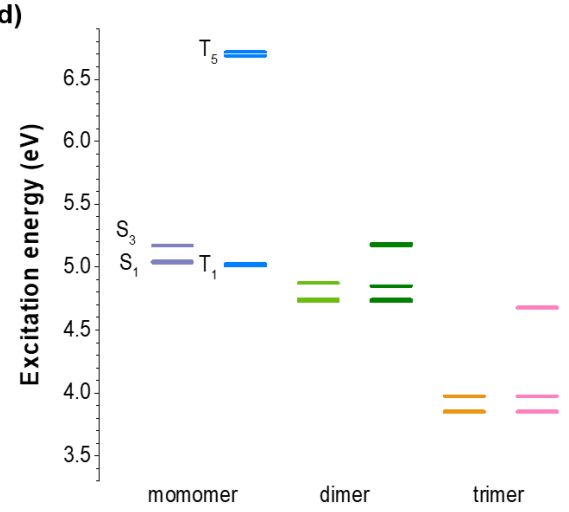

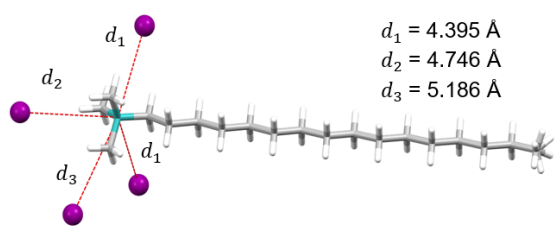

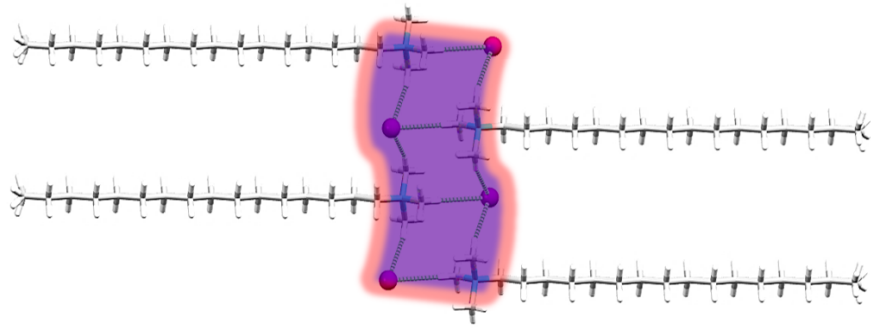

(e)

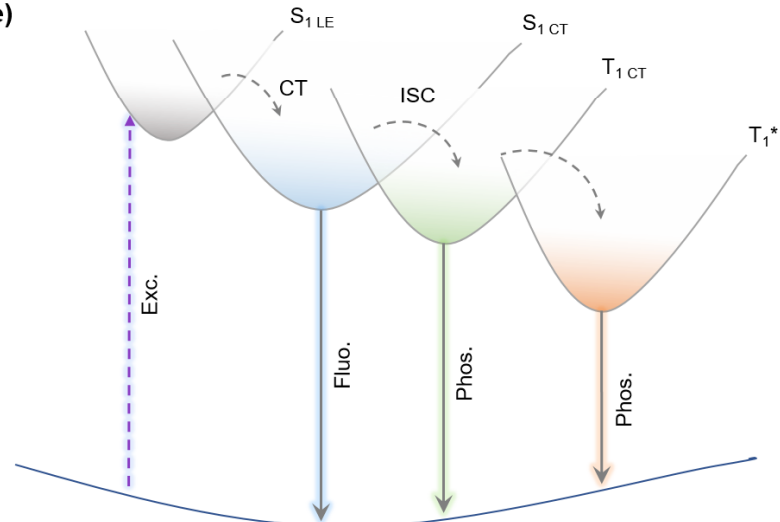

Figure 5. Intriguing emission of CTAI crystals under UV C irradiation $(220 \sim 260 \mathrm{~nm})$ and the emission mechanism understanding of QASs. (a) Excitation-PL mappings of CTAI crystals at room temperature. (b) Single crystal structure showing surrounding iodine anions around one quaternary ammonium cation and fragmental trimer packing of CTAI. (c) Electron density distributions of HOMO and LUMO levels and (d) energy levels of monomer, dimer and trimer of CTAI. (e) Proposed emission mechanism of QASs emphasizing charge transfer and energy transfer in diverse clustered ion pairs. 


\section{Physical blending and mechanical stimuli mediated emission}

Considering their outstanding PL tunability, these compounds are promising as smart materials for multimode advanced anticounterfeiting. ${ }^{[48-51]}$ As illustrated in Fig 6, an "emissive palette" was constructed with two complementary emissions at blue from CTAC and orange from CTAI $\left(\lambda_{\mathrm{ex}}=254\right.$ $\mathrm{nm})$. In detail, a series of powders with different PL were prepared by mixing and grinding CTAI and CTAC solids with varying proportions (Fig 6a). XRD diffractograms for the ground powders show sharp and well-resolved peaks (Fig 6b), suggesting their predominantly crystalline nature even upon vigorous mechanical grinding. Such stable crystal lattices should be ascribed to the powerful ionic bonding and remarkable hydrophobic interactions, which empowers bright and robust p-RTP afterglows from fully ground samples of QASs (Fig 6a). Utilizing these colorful ground powders, a bird pattern was painted (Fig 6c). As shown in Fig 6d, under 254 nm UV irradiation, an emissive bird consisting of CTAI (pink) and CTAC solids (blue), as well as their 1:1 (white) and 1:8 (blue-white) ground mixtures can be clearly visualized, whereas a rather bluish-green and a blue bird are observed under 312 and $365 \mathrm{~nm}$ UV lights, respectively, demonstrating apparent color-tunability in response to $\lambda_{\text {ex. }}$ In addition, upon removal of the UV irradiations, a green or yellow pattern is obtained. Therefore, multicolor PL and afterglows have enabled the advanced anticounterfeiting of the QAS solids by simply switching the UV excitation. The relative PL intensities at $618 \mathrm{~nm}$ for CTAI powders and at 356/424 nm for CTAC solids $\left(\lambda_{\mathrm{ex}}=254 \mathrm{~nm}\right)$ are gradually enhanced with increased CTAI fraction (Fig 6e). Notably, 1/1 and 8/1 CTAI/CTAC solids show cool white and warm white light emissions with the CIE coordinates of $(0.30,0.29)$ and $(0.34,0.32)$ (Fig 6g), respectively. Besides, the component regulated p-RTP is also seen from the delayed PL spectra $\left(t_{\mathrm{d}}=1 \mathrm{~ms}\right)$ and corresponding CIE coordinates (Fig 6f,h and Fig S18, Table S2), from which the afterglow color from bluish-green to green together with emission maximum from 460 to $516 \mathrm{~nm}$ is noticed. These results definetly suggest the feasibility to tune the PL of QAS solids upon physical blending and consequent grinding. 


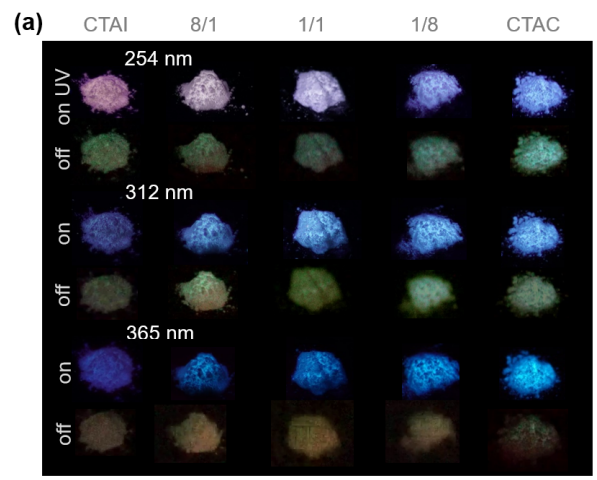

(b)

(c)
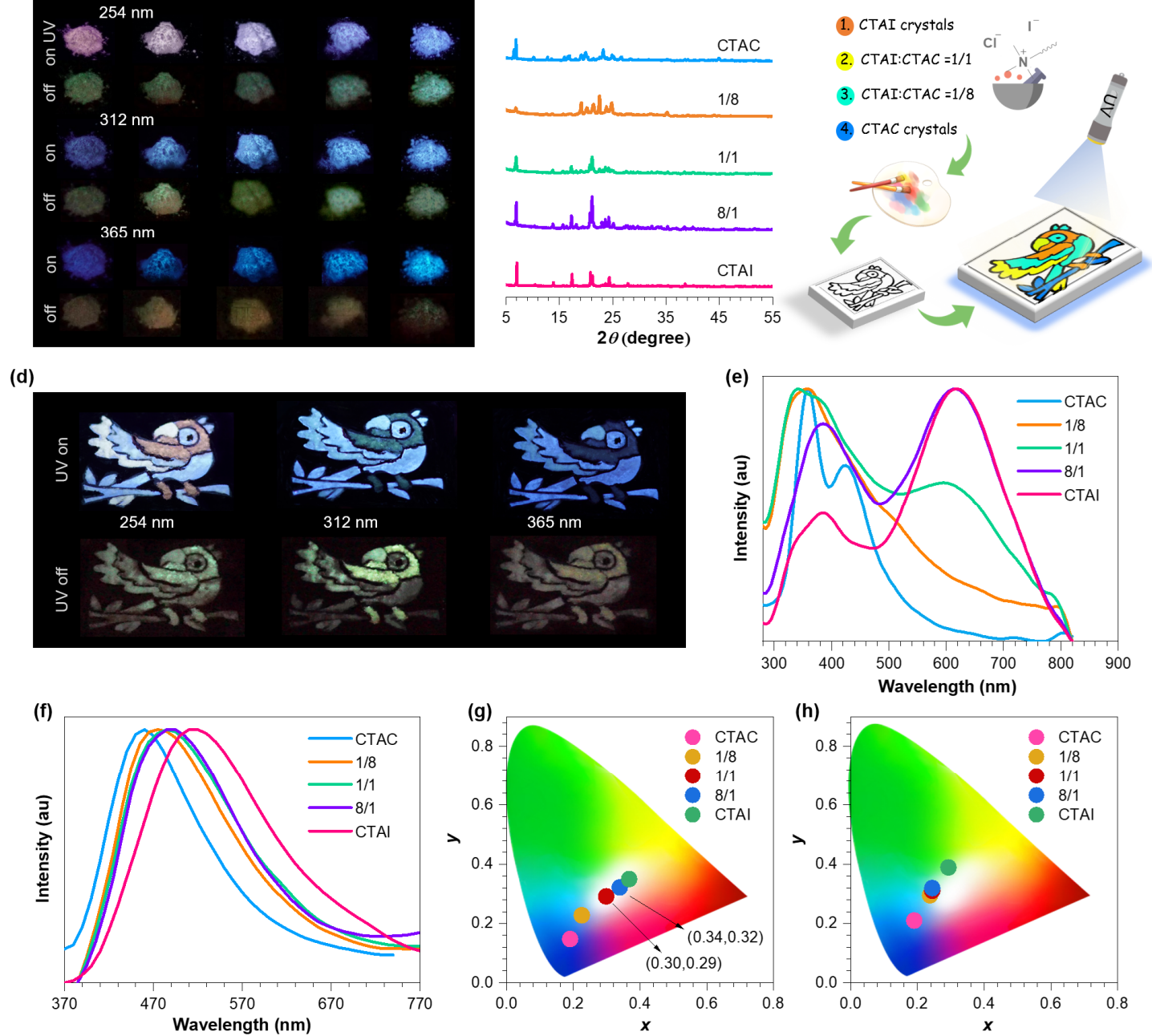

Figure 6. Tunable PL, white light emission and advanced multimode anticounterfeiting of physically blended solids of QASs. (a) Photographs of ground powders with different proportions of CTAI and CTAC taken under 254, 312 and $365 \mathrm{~nm}$ UV light or after ceasing the irradiations. (b) Powder XRD patterns of ground solids of CTAI, CTAC and their blends with different molar ratios of CTAI/CTAC. (c) Schematic illustration and (d) demonstration of the anticounterfeiting application based on color-tunable PL and p-RTP of different ground powders. $(\mathrm{e}, \mathrm{g})$ Prompt and $(\mathrm{f}, \mathrm{h})$ delayed $\left(t_{\mathrm{d}}=\right.$ $1 \mathrm{~ms})(\mathrm{e}, \mathrm{f})$ emission spectra and $(\mathrm{g}, \mathrm{h})$ their corresponding CIE coordinate diagrams of different ground powders upon excitation of $254 \mathrm{~nm}$ UV light.

\section{Summary and prospects}

In summary, we unveiled the general intrinsic emission from QASs, which were previously believed nonluminescent. The remarkable PL of QASs is attainable upon the clustering of ion pairs and charge 
transfer amongst the ions, which enable extended electronic delocalization and highly stiffened conformations. We observed fluorescence-phosphorescence dual emission in the crystals of QASs with $\Phi_{\mathrm{c}}$ and $\left\langle\tau>_{\mathrm{p}}\right.$ of up to $14.7 \%$ and $762 \mathrm{~ms}$, which can also be adjusted through the changes of alkyl chain length, excitation wavelength, temperature, counterion as well as physical blending and grinding, demonstrating effective PL regulation performance. Furthermore, impressive orange-red phosphorescence is obtained from CTAI crystals, probably stemming from charge transfer and energy transfer between diverse clusters. These refreshing PL properties of QASs shed new lights on researches regarding nonconventional luminophores, including underlying emission mechanism and promising design methodology. We have also demonstrated that QASs can be utilized in multimode advanced anticounterfeiting. We are optimistic that more QASs with much better PL performance and other functionalities could be rationally constructed. In brief, the prevailing intrinsic luminescence of QASs illustrated here may provide an alternative method for investigating the QAS related processes utilizing the PL technology and open previously unexplored opportunities for QASs in diverse disciplines.

\section{Acknowledgements}

This work was financially supported by the National Natural Science Foundation of China (51822303, 52073172), the Natural Science Foundation of Shanghai (20ZR1429400) and the "Shuguang Program" (20SG11) of Shanghai Education Development Foundation and Shanghai Municipal Education Commission.

\section{Reference}

[1] R. B. Restani, P. I. Morgado, M. P. Ribeiro, I. J. Correia, A. Aguiar-Ricardo and V. D. B. Bonifácio, Angew. Chem. Int. Ed. 2012, 51, 5162-5165.

[2] J. Pansieri, V. Josserand, S.-J. Lee, A. Rongier, D. Imbert, M. M. Sallanon, E. Kövari, T. G. Dane, C. Vendrely, O. Chaix-Pluchery, M. Guidetti, J. Vollaire, A. Fertin, Y. Usson, P. Rannou, J.-L. Coll, C. Marquette and V. Forge, Nat. Photonics 2019, 13, 473-479.

[3] W. I. Lee, Y. Bae and A. J. Bard, J. Am. Chem. Soc. 2004, 126, 8358-8359.

[4] D. Wang and T. Imae, J. Am. Chem. Soc. 2004, 126, 13204-13205. 
[5] D. A. Tomalia, B. Klajnert-Maculewicz, K. A. M. Johnson, H. F. Brinkman, A. Janaszewska and D. M. Hedstrand, Prog. Polym. Sci. 2019, 90, 35-117.

[6] H. Zhang, Z. Zhao, P. R. McGonigal, R. Ye, S. Liu, J. W. Y. Lam, R. T. K. Kwok, W. Z. Yuan, J. Xie, A. L. Rogach and B. Z. Tang, Mater. Today 2020, 32, 275-292.

[7] C. Shang, Y. Zhao, J. Long, Y. Ji and H. Wang, J. Mater. Chem. C 2020, 8, 1017-1024.

[8] L. Gu, H. Shi, L. Bian, M. Gu, K. Ling, X. Wang, H. Ma, S. Cai, W. Ning, L. Fu, H. Wang, S. Wang, Y. Gao, W. Yao, F. Huo, Y. Tao, Z. An, X. Liu and W. Huang, Nat. Photonics 2019, 13, 406-411.

[9] Y. Wang, S. Tang, Y. Wen, S. Zheng, B. Yang and W. Z. Yuan, Mater. Horiz. 2020, 7, $2105-$ 2112.

[10] M. Fang, J. Yang, X. Xiang, Y. Xie, Y. Dong, Q. Peng, Q. Li and Z. Li, Mater. Chem. Front. 2018, 2, 2124-2129.

[11] S. Wang, D. Wu, S. Yang, Z. Lin and Q. Ling, Mater. Chem. Front. 2020, 4, 1198-1205.

[12] Z.-F. Liu, X. Chen and W. J. Jin, J. Mater. Chem. C 2020, 8, 7330-7335.

[13] H. Zheng, P. Cao, Y. Wang, X. Lu and P. Wu, Angew. Chem. Int. Ed. 2021, 60, 9500-9506.

[14] M. Singh, K. Liu, S. Qu, H. Ma, H. Shi, Z. An and W. Huang, Adv. Optical Mater. 2021, 9, 2002197.

[15] R. Kabe and C. Adachi, Nature 2017, 550, 384-387.

[16] M. Sun, C.-Y. Hong and C.-Y. Pan, J. Am. Chem. Soc. 2012, 134, 20581-20584.

[17] W. Yang and C.-Y. Pan, Macromol. Rapid Commun. 2009, 30, 2096-2101.

[18] L. Pastor-Pérez, Y. Chen, Z. Shen, A. Lahoz and S.-E. Stiriba, Macromol. Rapid Commun. 2007, 28, 1404-1409.

[19] S. Tao, S. Zhu, T. Feng, C. Zheng and B. Yang, Angew. Chem. Int. Ed. 2020, 59, 9826-9840.

[20] Y. Feng, T. Bai, H. Yan, F. Ding, L. Bai and W. Feng, Macromolecules 2019, 52, 3075-3082.

[21] E. Zhao, J. W. Y. Lam, L. Meng, Y. Hong, H. Deng, G. Bai, X. Huang, J. Hao and B. Z. Tang, Macromolecules 2015, 48, 64-71.

[22] Wu, Y. Liu, He and S. H. Goh, Macromolecules 2005, 38, 9906-9909.

[23] K. Noboru, I. Shin-ichiro and T. Shigeo, Chem. Lett. 1983, 12, 455-458.

[24] R. J. Hartley and L. R. Faulkner, J. Am. Chem. Soc. 1985, 107, 3436-3442. 
[25] Q.-X. Mei, L. Lai, Z.-Q. Zhou, P. Mei, Z.-H. Ren, Y.-C. Zheng and Y. Liu, Soft Matter 2017, $13,7273-7282$.

[26] N. Jiang, D. Zhu, Z. Su and M. R. Bryce, Mater. Chem. Front. 2021, 5, 60-75.

[27] L. Xu, J. Cao, S. Zhong, Y. Gao and X. Cui, J. Agric. Food Chem. 2021, 69, 7680-7686.

[28] X. Li, M. Li, M. Yang, H. Xiao, L. Wang, Z. Chen, S. Liu, J. Li, S. Li and T. D. James, Coord. Chem. Rev. 2020, 418, 213358.

[29] W. Z. Yuan and Y. Zhang, J. Polym. Sci. Polym. Chem. 2017, 55, 560-574.

[30] Y. Gong, Y. Tan, J. Mei, Y. Zhang, W. Yuan, Y. Zhang, J. Sun and B. Z. Tang, Sci. China Chem. 2013, 56, 1178-1182.

[31] Q. Zhou, B. Cao, C. Zhu, S. Xu, Y. Gong, W. Z. Yuan and Y. Zhang, Small 2016, 12, 65866592.

[32] T. Liu, X. Pan, X. Meng, Y. Liu, D. Wei, W. Ma, L. Huo, X. Sun, T. H. Lee, M. Huang, H. Choi, J. Y. Kim, W. C. H. Choy and Y. Sun, Adv. Mater. 2017, 29, 1604251.

[33] T. Zhang, X. Ma, H. Wu, L. Zhu, Y. Zhao and H. Tian, Angew. Chem. Int. Ed. 2020, 59, 1120611216.

[34] I. Bhattacharjee and S. Hirata, Adv. Mater. 2020, 32, 2001348.

[35] Y. Tian, J. Yang, Z. Liu, M. Gao, X. Li, W. Che, M. Fang and Z. Li, Angew. Chem. Int. Ed. 2021, $60,20259$.

[36] W. Zhao, Z. He and B. Z. Tang, Nat. Rev. Mater. 2020, 5, 869-885.

[37] J. Yang, M. Fang and Z. Li, Aggregate. 2020, 1, 6-18.

[38] H.-J. Yu, Q. Zhou, X. Dai, F.-F. Shen, Y.-M. Zhang, X. Xu and Y. Liu, J. Am. Chem. Soc. 2021, $143,13887-13894$.

[39] Y. Shi, R. Lei, F. Li, S. Li, X. Tao, P. Yang, X. Chen, Q. Zhao and Z. L. Wang, ACS Energy Lett. 2021, DOI: 10.1021/acsenergylett.1c01508.

[40] J. Zhang, S. Xu, Z. Wang, P. Xue, W. Wang, L. Zhang, Y. Shi, W. Huang and R. Chen, Angew. Chem. Int. Ed. 2021, 60, 17094-17101.

[41] B. Chen, W. Huang, X. Nie, F. Liao, H. Miao, X. Zhang and G. Zhang, Angew. Chem. Int. Ed. 2021, 60, 16970-16973. 
[42] Y. Lei, W. Dai, J. Guan, S. Guo, F. Ren, Y. Zhou, J. Shi, B. Tong, Z. Cai, J. Zheng and Y. Dong, Angew. Chem. Int. Ed. 2020, 59, 16054-16060.

[43] E. Lucenti, A. Forni, C. Botta, L. Carlucci, C. Giannini, D. Marinotto, A. Pavanello, A. Previtali, S. Righetto and E. Cariati, Angew. Chem. Int. Ed. 2017, 56, 16302-16307.

[44] M. Shimizu and T. Sakurai, ChemPlusChem 2021, 86, 446-459.

[45] S. Zheng, T. Zhu, Y. Wang, T. Yang and W. Z. Yuan, Angew. Chem. Int. Ed. 2020, 59, 1001810022.

[46] Z. Yang, C. Xu, W. Li, Z. Mao, X. Ge, Q. Huang, H. Deng, J. Zhao, F. L. Gu, Y. Zhang and Z. Chi, Angew. Chem. Int. Ed. 2020, 59, 17451-17455.

[47] O. Bolton, K. Lee, H.-J. Kim, K. Y. Lin and J. Kim, Nat. Chem. 2011, 3, 205-210.

[48] K. Jiang, Y. Wang, X. Gao, C. Cai, H. Lin, Angew. Chem. Int. Ed. 2018, 57, 6216-6220.

[49] Kenry, C. Chen, B. Liu, Nat. Commun. 2019, 10, 2111.

[50] M. Louis, H. Thomas, M. Gmelch, A. Haft, F. Fries and S. Reineke, Adv. Mater. 2019, 31, 1807887.

[51] Y. Zhang, Z. Wang, Y. Su, Y. Zheng, W. Tang, C. Yang, H. Tang, L. Qu, Y. Li and Y. Zhao, 2021, 2021, 8096263. 